\title{
IJWHR \\ A Mirror Intervention for the Postmastectomy Mirror Experience
}

\author{
Wyona M. Freysteinson*
}

$\mathrm{T}$ Through my research, I have come to understand that women who have undergone a significant body change due to trauma, surgery, or disease find viewing their bodies in a mirror is an emotionally charged, distressing experience. In this editorial, I focus on the experience of viewing self in the mirror postmastectomy, a nursing education intervention gleaned from an understanding of this experience, and this debate: Should we wait on randomized control trials (RCTs) prior to implementing interventions for the mirror experience for those women who will have a mastectomy OR is this a case in which we should abandon the hierarchy of evidence?

Evidence-based practice (EBP) has had a tremendous impact of the quality of nursing care. In EBP, there is an emphasis on "identifying the best available research evidence and integrating it" (1). In order to determine the best literature, evidence hierarchies are used where RCTs and systematic reviews of RCTs are at the top of the hierarchy. As such, there is a great emphasis on this type of evidence. However, Polit and Beck (1) stress RCTs are not always appropriate for all clinical questions. I would argue that for women who have had a mastectomy with or without reconstructive surgery that RCTs are not necessarily needed in order to give compassionate nursing care and support toward the postmastectomy mirror experience. Many years ago, a terminally ill woman shared with me in a phenomenological research study that when she returned home from the hospital after having a mastectomy, the first place she went was into her bathroom to look at her incision for the first time. She said that when she viewed her reflection in the mirror, "she felt like running out on the road and screaming" (2). Knobf's theory of Carrying On - provides a common - sense framework for my work. Knobf theorized that if people with cancer are prepared for the experiences they will endure, they will better withstand those experiences (3).

In a study, 12 women 3-12 months postmastectomy shared their mirror experiences in audio-taped interviews. These women indicated they had joined the study so that other women would find help in the future in viewing self in the mirror after a mastectomy. A model of the mirror experience was developed and decision, seeing, and consent were found to be key moments within the experience (4). There were three reasons why women viewed themselves in the mirror: personal grooming, some women discovered the mirror was a useful tool when caring for their incisions and drains, and curiosity. This curiosity was often accompanied by feelings of dread and fear. In other words, women lived a paradoxical experience of wanting/not wanting to see their mastectomy sites in a mirror.

The second element in this model is seeing: women see in a mirror in three ways: with the eyes, with the mind's eye, and with the mind (i.e. I see what this means to me). One woman with an eye disease realized she needed a magnified mirror to help see with her eyes. Seeing with the mind's eye is anticipating what one may see in the mirror. Two women who had simple mastectomies in the past anticipated and hoped that the second mastectomy incision would be similar to their current scars so that their chests would appear symmetrical. Both women were shocked and angered to see radical mastectomy incisions (this topic in and of itself needs further exploration). One young woman spent hours on Google reviewing mastectomy pictures prior to her surgery. She said she put herself through unnecessary agony for days as she avoided looking in a mirror because she anticipated she would have a large ugly incision. She was surprised to find she had a simple mastectomy incision. One sees or understands

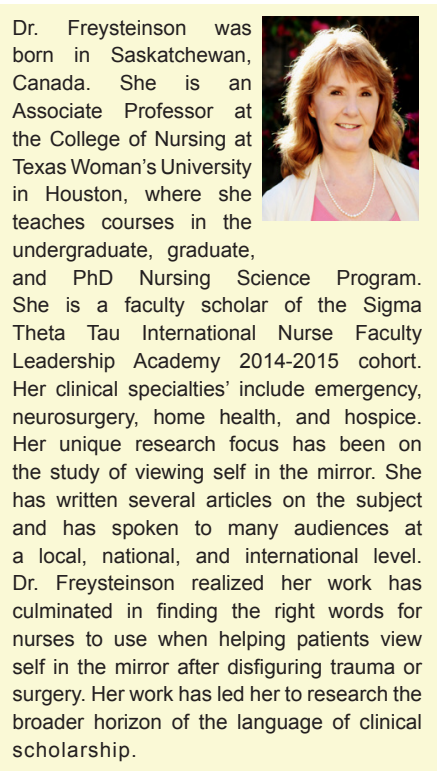

Dr. Freysteinson was

Canada. She is an Associate Professor at Texas Woman's University

She is a faculty scholar of the Sigma Leadership Academy 2014-2015 cohort. neurosurgery, home health, and hospice. the study of viewing self in the mirror. She has written several articles on the subject local, national, and international level. Dr. Freysteinson realized her work has
culminated in finding the right words for al . 
the reflection seen in the mirror. This moment of understanding is unique to each individual's way of being in the world. This understanding may be accompanied by emotions of shock, denial, fear, anger, sadness or one may feel relieved. The third element is consent. Consent to what one has seen in the mirror was accompanied by great suffering for all participants: One woman said she sat in her bathtub for hours crying after seeing her mastectomy site in the mirror (4).

A randomized control pilot study was conducted to study the feasibility of a mirror program to prepare women for a mastectomy. Pre and postoperative data was collected from 10 subjects and 9 control subjects. The intervention used was performed by oncology nurse navigators (ONNs). These nurses were chosen for the study as ONNs see patients prior to and after surgery. Each subject meant with her ONN prior to surgery. The ONNs explained what the incision and drains would look like postoperatively. An example from the ONN educational script is:

You may want to view your mastectomy site with your physician, nurse, and/or loved one. Alternatively, you may choose to view your mastectomy site alone. When you view yourself for the first time, you may feel shocked, mad, or sad because the incision does not look like the picture you had in your mind.

The ONN's talked with each woman about their fears, concerns, and plans to view self in the mirror. Although this was a feasibility study, there was a trend noted that the intervention group body image (BIS) and well-being (SF-36) scores improved, and the control group BIS and SF-36 worsened (5).

In our healthcare world with its emphasis on the need for the best research, this work would require larger RCTs in order for this intervention to be put into practice. We may want to know if there may be a reduction in postoperative infections if woman used mirrors to aid in assessing their mastectomy incisions. In terms of body image, we may want to study the effect of nursing mirror support on psychological outcomes. However, the qualitative studies that we have on this issue provide, I believe, adequate evidence that the postoperative mirror experience for these women is desperately in need of nursing support. Perhaps we need to reconsider or redefine the hierarchy of evidence for those extremely distressing patient experiences we encounter or discover?

With research dollars at a premium, do nurses really need more research in order to provide this intervention? On one hand, I know that with our current emphasis on EBP and the hierarchy of evidence, this intervention may be lost or ignored unless it is accompanied by larger RCTs. On the other hand, I believe it would be nice to know if this particular intervention may reduce postoperative infection or improve long-term psychological outcomes. However, I do not believe this hierarchy of evidence is necessary in order to prepare women for the immediate experience of viewing self in the mirror after the amputation of a breast. I believe nurses can help women enter into the mirror experience so that each woman may have a basic understanding of the experience and she may know, at least, that "my nurse understands what I am going through". I welcome a continuing dialogue on this subject.

\section{Ethical issues}

The Institutional Review Board reviewed and approved the study prior to participant recruitment and data collection.

\section{Financial support}

This study was funded by a substantial grant from The Houston Endowment, USA.

\section{Conflict of interests}

The authors have no conflict of interests to disclose.

\section{Acknowledgements}

We appreciate the unflagging assistance and support of the administrators and staff of the five shelters in Harris County and the Chief and staff of The Harris County District Attorney's Office, Family Criminal Law Division.

\section{References}

1. Polit FP, Beck CT. Nursing research: generating and assessing evidence for nursing practice. 9th ed. LWW; 2012.

2. Freysteinson WM. The lived experience of terminally ill women viewing self in the mirror [unpublished master's thesis]. 1994.

3. Knobf MT. Being prepared: essential to self-care and quality of life for the person with cancer. J Clin Oncol. 2013;17:255-261. doi: 10.1188/13.CJON.255-261.

4. Freysteinson WM, Deutsch AS, Lewis C, Sisk A, Wuest L, Cesario S K. The experience of viewing self in the mirror after a mastectomy. Oncol Nurs Forum. 2012;39:361-369. doi: 10.1188/12.ONF.361-369.

5. Freysteinson WM, Deutsch A, Davin K, et al. The mirror program: preparing women for the postoperative mirror viewing experience. Nurs Forum. 2014. doi: 10.1111/nuf.12108

Copyright $\odot 2015$ The Author(s); This is an open-access article distributed under the terms of the Creative Commons Attribution License (http://creativecommons.org/licenses/by/4.0), which permits unrestricted use, distribution, and reproduction in any medium, provided the original work is properly cited. 